\title{
Enseigner une matière disciplinaire en langue étrangère dans le contexte français des sciences sociales : défi, observations et implications
}

Gail Taillefer

\section{(2) OpenEdition}

Journals

Édition électronique

URL : http://journals.openedition.org/asp/884

DOI : $10.4000 /$ asp.884

ISBN : 978-2-8218-0398-5

ISSN : 2108-6354

Éditeur

Groupe d'étude et de recherche en anglais de spécialité

Édition imprimée

Date de publication : 1 décembre 2004

Pagination : 111-126

ISSN : 1246-8185

Référence électronique

Gail Taillefer, «Enseigner une matière disciplinaire en langue étrangère dans le contexte français des sciences sociales : défi, observations et implications », ASp [En ligne], 45-46 | 2004, mis en ligne le 02 mars 2010, consulté le 19 avril 2019. URL : http://journals.openedition.org/asp/884 ; DOI : 10.4000/ asp.884

Ce document a été généré automatiquement le 19 avril 2019

Tous droits réservés 


\title{
Enseigner une matière disciplinaire en langue étrangère dans le contexte français des sciences sociales : défi, observations et implications
}

\author{
Gail Taillefer
}

\section{Introduction}

1 Enseigner une matière disciplinaire en langue étrangère se pratique dans l'enseignement supérieur de nombreux pays sous différentes formes et dans divers contextes: un programme entier ou seulement quelques matières, avec sélection ou non des apprenants selon leur compétence linguistique, avec des apprenants et des enseignants de langues et de cultures variées dans des disciplines multiples. Cet enseignement existe également sous différents noms : en français, EMILE (enseignement d'une matière par l'intégration d'une langue étrangère), ECI (enseignement à contenu intégré) ; en anglais, TTFL (teaching through a foreign language), CLIL (content and language integrated learning). Langue et contenu sont toujours intégrés, mais selon chaque contexte le poids de chaque facteur varie. Dans la tradition de la langue de spécialité en anglistique, c'est la langue qui prédomine (Mémet 2001) ; dans les autres disciplines, la langue étrangère joue souvent un rôle utilitaire et son apprentissage est secondaire, « incidental » (Hellekjaer \& Westergaard 2003 : 66). Plus rares, et plus positifs, semblent être les cas où l'objectif est réellement double - disciplinaire aussi bien que linguistique (Hellekjaer \& Wilkinson 2003 : 86, 93 ; Wolff 2003 : 37 ; Stoller \& Grabe 1997).

2 La question de cet équilibre se pose car cet enseignement / apprentissage plus ou moins à double « foyer » se généralise en France, impulsé par la Déclaration de Bologne favorisant la mobilité des étudiants. Mémet (2003: 139) donne quelques exemples de nouveaux 
cadres de l'EMILE; Orlac'h (2005) recense plus de 300 cursus en anglais par rapport à la dizaine d'il y a quelques années. Mais il en précise que l'offre est limitée en comparaison avec l'Allemagne, où 1000 cursus en anglais sont répertoriés. Le phénomène est très développé en Europe du nord depuis plusieurs années (Hellekjaer \& Westergaard 2003, Hellekjaer \& Wilkinson 2003).

\section{Enseignement à contenu intégré : fondements, mises en garde}

\subsection{Fondements}

3 Le bien-fondé de l'EMILE n'est plus à prouver. Grabe et Stoller (1997) rappellent les différents courants de la recherche qui étayent cette approche : second language acquisition (comprehensible input, importance de output, approches socioculturelles, cognitive academic language proficiency), training studies (cooperative learning, strategy instruction, lecture extensive), psychologie cognitive (depth-of-processing, traitement du discours, motivation, expertise), et enfin les résultats positifs observés dans des programmes d'EMILE. Ces auteurs expliquent les bénéfices de l'EMILE par l'importance de l'exposition à la langue étrangère et son utilisation, par la pertinence de sa contextualisation, par l'accroissement de la motivation et par les possibilités ouvertes à une réflexion métacognitive sur l'apprentissage (1997 : 19-20). Plus récemment, Wolff (2003) montre comment les théories interactionnistes de la recherche en acquisition de la langue seconde (second language acquisition) et le constructivisme peuvent expliquer le haut niveau d'apprentissage linguistique et disciplinaire qui résulte d'une approche intégrée. Pour lui, les facteurs-clés sont les notions de input pertinent, d'interaction impliquée et de construction de savoir et de savoir-faire. Enfin, pour Marsh, Marsland et Stenberg (2001:13-14), « using languages to learn in order to learn to use languages » - même quand cela ne représente que $10 \%$ d'un cursus - est bénéfique en termes personnels (confiance en soi, motivation), linguistiques (compétence communicative) et disciplinaires.

\subsection{Mises en garde}

4 Face à une probable multiplication des cursus en anglais dans l'enseignement supérieur en France, la question des enjeux se pose. La recherche en LANSAD occupe une place privilégiée pour y répondre et peut jouer un rôle de sensibilisation et d'information. Cette recherche se fonde forcément sur le terrain, à partir d'enquêtes et d'observations auprès des acteurs : étudiants, enseignants et administrateurs. Marsh et al. (2001) préconisent un bilan complet comprenant plusieurs paramètres: l'environnement institutionnel (contexte local, état d'esprit, ressources humaines, cadre académique), la mise en œuvre pratique (curriculum, travail en équipe, compétence linguistique des enseignants, styles d'enseignement / apprentissage, évaluation du programme) et la perception de l'EMILE dans un cadre plus large (développement institutionnel, travail en réseaux, lien enseignement-entreprise). Mais, d'après Hellekjaer et Westergaard (2003: 77), peu de programmes d'EMILE sont conçus avec autant de prévision et d'investissement.

5 Les écueils sont pourtant nombreux et se concentrent sur la divergence entre, d'une part, les compétences, les perceptions, les attentes - et donc les pratiques - des étudiants et des enseignants et, d'autre part, la reconnaissance explicite de ces facteurs par les différents 
acteurs. Marsh et al. (2001) soulignent la nature holistique de l'enseignement / apprentissage et le fait que, dans l'EMILE, on ne peut pas séparer les compétences linguistiques des compétences pédagogiques. Mais, pour éviter ces écueils, il faut clairement les repérer. La littérature identifie deux grandes lignes d'analyse: la compétence linguistique et la notion de culture. Cette dernière comprend les notions de culture disciplinaire, de culture sociétale ou ethnique et de culture didactique.

\subsubsection{Compétences linguistiques}

6 La question des compétences linguistiques est pertinente pour les enseignants comme pour les étudiants. Chez les premiers, Kurtán (2003: 147-150) relève surtout la compétence communicative ou le facteur «d'aisance ». Si l'on est à l'aise avec la langue disciplinaire (lexique, discours, genre), y a-t-il des difficultés de prononciation, de fluidité, de clarté d'expression, de vocabulaire et de tournures variées pour pouvoir reformuler ? Hellekjaer et Westergaard (2003: 76) rapportent un taux « considérable » de demande de soutien linguistique (environ $45 \%$ chez les enseignants dans leur enquête sur 58 programmes scandinaves). Marsh et al. (2001: 146) posent d'emblée la question : « How good is good enough?» et ajoutent que certaines matières exigent des compétences linguistiques plus développées que d'autres, notamment les sciences humaines ou sociales - plus conceptuelles - par rapport aux sciences exactes.

7 S'il existe des tests de compétence pour certifier un niveau en langue générale, académique et professionnelle, il n'y en a pas pour l'enseignement d'une discipline en langue étrangère. Le Cadre européen commun de référence est évidemment la nouvelle ressource dans la matière, mais Marsh et al. (2001: 149) soulignent qu'on peut être opérationnel sans être parfait et qu'il est tout à fait acceptable que les étudiants aient un meilleur niveau linguistique que l'enseignant, à condition que règne un climat de confiance, soutenu par un travail commun pour atteindre les objectifs.

Chez les étudiants, il est plus facile, au moyen d'examens ou de tests calibrés, d'exiger un certain niveau de compétence linguistique, mais tous les programmes d'EMILE ne le font pas, se contentant d'une évaluation externe plus floue (notes de langue, recommandation) ou d'une auto-évaluation. Le résultat est plus ou moins heureux selon les cas. Dans leur étude, Hellekjaer et Westergaard (2003: 76) mentionnent un taux moyen de 54,5\% de demandes de soutien linguistique, précisant qu'il est plus fort dans les établissements où le recrutement des étudiants comprend des candidats nationaux. Confrontés à des difficultés linguistiques chez leurs étudiants, ayant pour conséquence le bavardage ("Qu'est-ce qu'il a dit?») et l'inattention, des enseignants expérimentés disent surtout essayer de simplifier leur discours. Ils ralentissent le débit, évitent des mots complexes ou rares et s'appuient davantage qu'en L1 sur un soutien visuel (polycopié, rétroprojection ou vidéoprojection) (Flowerdew \& Miller 1996: 129-134). Le risque en $\mathrm{L} 2$ est donc de moins approfondir le sujet, d'encourager le copiage irréfléchi ou une compréhension trop facile limitée aux seules informations écrites. Mais quoi qu'il en soit, Hellekjaer et Wilkinson (2003: 88-89) insistent sur l'importance d'expliciter les attentes linguistiques, notamment celles concernant l'expression écrite, ainsi que l'envergure envisagée des programmes d'EMILE. En effet, ces auteurs signalent davantage de difficultés linguistiques là où le développement des programmes d'EMILE est plus important (Hellekjaer \& Westergaard $2003:$ 77). 


\subsubsection{Compétences interculturelles}

9 La deuxième grande catégorie d'écueils relève du domaine interculturel. La recherche décèle des problèmes aux niveaux de la culture disciplinaire (et de son rapport avec la langue), de la culture sociétale ou ethnique et de la culture didactique.

Chaque discipline a ses propres théories, concepts, normes et structures de pensée et ceux-ci s'expriment au moyen de discours (genres), de structures linguistiques et de terminologies spécifiques (Flowerdew \& Miller 1995 : 366). L'EMILE en droit peut donc différer de l'EMILE en économie ou en gestion. Flowerdew et Miller (366-367) comparent un cours de droit fondé sur une série de tâches de résolution de problèmes destinées à illustrer un concept juridique à un cours d'économie structuré autour d'une série de concepts illustrés par des exemples, et à un cours de gestion comparant différents modèles ou systèmes. Si le juriste commence par des définitions, l'ingénieur (ou l'économiste) commence par des graphiques et des tableaux.

11 De la même manière, les écrits universitaires varient d'une culture disciplinaire à l'autre (Swales 1990), à quoi s'ajoute dans l'EMILE le filtre de la rhétorique contrastive (Kaplan 1966, 1995 ; Ulijn \& Salager-Meyer 1998). Le métatexte économique en espagnol et en anglais n'est pas le même (Valero-Garcés 1996), mais les deux diffèrent profondément du style narratif et d'articulation complexe d'un Law Report anglais et de la jurisprudence française de nature dense et aride (Field 199911). La même différence de culture disciplinaire, et en même temps linguistique, se voit dans l'évaluation universitaire. Les pratiques traditionnelles varient fortement, par exemple, entre la France et l'Angleterre dans les études de droit. Si les étudiants français sont évalués surtout au moyen d'examens écrits ou oraux, leurs camarades anglais le sont généralement sur la base de travaux de recherche personnelle en même temps que d'examens écrits. Les étudiants en économie, en France, passent des écrits traditionnels (et plus rarement des oraux), alors que les étudiants anglais ont plus souvent des recherches à faire et des QCM à passer (Taillefer, à paraître).

12 La culture sociétale a également, bien évidemment, son importance dans l'EMILE : selon les contextes, les enseignants et les étudiants viennent de pays et de langues maternelles et secondes variés. Plusieurs auteurs s'inspirent de la grille de lecture proposée par Hofstede (1991) pour cerner les situations de choc culturel : l'importance de la hiérarchie et de l'autorité (" power distance »), individualisme ou collectivisme, le niveau de tolérance de l'imprévu ("uncertainty avoidance») et l'affirmation de soi, ou s'occuper plutôt de l'autre («masculinity-femininity»). Kurtán (2003), Isani et Andreolle (2001), et surtout Flowerdew et Miller (1995), dans le contexte de professeurs britanniques enseignant en anglais à Hong Kong, font part des difficultés rencontrées aux niveaux des valeurs, des attentes et des modèles de comportement divergents entre enseignants et apprenants : le plagiat, par exemple, vu comme expression de respect de l'autorité des experts; la " triche ", vue comme effort de solidarité collective ; le besoin de communiquer - ou de recevoir - des attentes explicites pour éviter l'incertitude ; un enseignement focalisé sur le cours magistral ou reconnaissant également les besoins des apprenants.

Les aspects de la culture sociétale influencent très directement la politique institutionnelle et la didactique. D'abord, jusqu'à quel point la culture institutionnelle valorise-t-elle réellement l'acquisition d'une compétence en langue étrangère ? Ensuite, dans une culture donnée, le rang social de l'enseignant lui permet-il facilement de se 
remettre en question dans une situation nouvelle telle que l'EMILE ? Culturellement, en ressent-il même le besoin (Kurtán 2003 : 149) ? Si oui, et s'il peut « avouer » d'éventuelles difficultés linguistiques, y a-t-il une tradition de développement professionnel ? Quel est son rôle en tant qu'enseignant: transmettre un savoir (à reproduire), ou aider les apprenants à construire le leur (Flowerdew \& Miller 1996 :124-125 ; Kurtán 2003 : 148-9) ? Ses attentes pédagogiques (travail personnel exigé, nature de l'évaluation) doivent-elles être explicitées? Comment voit-il son potentiel de guide linguistique en plus de son rôle scientifique ? Isani et Andréolle (2001 : 231) se demandent jusqu'où peut (ou doit) aller un enseignant dans la redéfinition de sa propre culture didactique pour aider à atteindre les objectifs pédagogiques des apprenants d'une culture didactique éloignée.

Chez les étudiants, la culture sociétale se traduit en termes pédagogiques de la même manière. Quelle niveau d'interactivité avec l'enseignant est-il «normal» et quel degré d'initiative et d'investissement personnels? Peut-on ne pas partager l'opinion de l'enseignant? Quelle attitude avoir par rapport au niveau de performance linguistique : faut-il viser la perfection? Quelle fourchette de notes est-elle « honorable » et, au besoin, peut-on la négocier?

列 vaut bien la "chandelle» de multiples enjeux, quels sont les risques potentiels si certaines précautions ne sont pas prises? Hellekjaer et Wilkinson (2003 : 99) indiquent que la qualité d'apprentissage pourrait en souffrir. Les plus jeunes étudiants, insuffisamment mûrs et moins compétents que leurs aînés en langue étrangère, ont besoin de davantage de temps qu'en langue maternelle pour atteindre les mêmes objectifs. L'EMILE pourrait-il créer une "fracture " linguistique - et donc un système officieux à deux vitesses - entre les étudiants ou entre les enseignants au sein d'un même établissement français offrant des diplômes nationaux ? Et pire, si le bilan n'est pas satisfaisant, n'y a-t-il pas le risque de discréditer l'enseignement des langues étrangères, vu parfois comme quelque chose qui prend " trop de place ", coûte trop cher et complique les emplois du temps (Isani \& Andréolle $2001: 232$ ) ?

\subsection{Questions de recherche}

Si l'EMILE est donc une arme à double tranchant, nous avons voulu observer sa mise en œuvre dans une université française de sciences sociales. Serait-il une réussite? Des difficultés linguistiques ou culturelles surgiraient-elles chez les enseignants et chez les étudiants? Verrait-on des pistes pour les étudiants plus jeunes qui souhaiteraient pouvoir participer à cet EMILE ? Enfin, y aurait-il un nouveau rôle pour le Département des langues et civilisations au sein de l'université, rôle de médiation, de remédiation, de travail parallèle?

À cette dernière question, la littérature répond par plusieurs modèles avec une échelle d'implication variable. Dudley-Evans et St. John (1998 : 41-48) distinguent trois niveaux : coopération, où l'enseignant de langues prend l'initiative en enquêtant sur la place des langues dans le curriculum; collaboration, où les enseignants des deux disciplines travaillent ensemble pour préparer leurs étudiants à des but définis; enseignement en équipe, où les deux enseignants partagent la salle de cours. Hellekjaer et Wilkinson (2003 : 92) proposent également trois schémas : cours de préparation (souvent peu motivants car distincts), cours parallèles, associés ou non aux cours disciplinaires, cours adjunct, réellement intégrés aux cours disciplinaires. Ces derniers visent les compétences nécessaires "pour maîtriser le contenu disciplinaire» (84) et se déroulent dans un 
environnement interactif, fondé sur le vocabulaire et la terminologie, l'analyse du discours et les compétences stratégiques pour la lecture et l'expression écrite. Enfin, Teemant, Bernhardt et Rodríguez-Muñoz (1997) soulèvent deux questions «miroirs": que doivent savoir les enseignants disciplinaires sur les besoins linguistiques de leurs étudiants et quelles stratégies les enseignants de langues peuvent-il fournir aux collègues disciplinaires ? La réponse demande de la diplomatie :

How directly you approach collaboration depends on your school's readiness to recognize the needs of ESL students [... It means] negotiating a protocol for effective collaboration among ESL and content-area faculty. (Teemant, Bernhardt et Rodríguez-Muñoz 1997 : 318).

Wolff (2003: 36) demande comment on peut organiser l'environnement d'enseignement / apprentissage de CLIL de manière optimale. Les auteurs déjà cités offrent un certain nombre de pistes, mais Stoller et Grabe (1997) suggèrent une vision composite des meilleurs modèles. Leur approche se fonde sur «theme, text, topics (sous-thèmes), threads (liens thématiques), tasks, et transitions" (entre les thèmes / sujets). Un autre point d'entrée, et sans doute la clé de voûte, est le corps des enseignants prêts à relever ce défi. Une professeure franco-britannique de naissance et de vie, fréquemment invitée à l'université Toulouse 1 pour enseigner le droit en anglais, cerne bien les enjeux du contexte particulier de l'EMILE et nous permet d'ouvrir le débat local. Elle fait la part des cours intensifs donnés en France par rapport à son enseignement extensif en Angleterre, des étudiants spécialistes en droit par rapport à des spécialistes d'autres disciplines, d'un public pour qui l'anglais est une L2 ou une L3 ou L4 (et dont le niveau peut être faible, rendant l'interaction difficile et l'utilisation de transparents essentielle), des étudiants de culture sociétale européenne ou non européenne nécessitant un choix judicieux d'exemples parlants et des analogies faisant appel à différentes cultures pour permettre des comparaisons. Enfin, ses méthodes d'évaluation font moins appel à la mémoire qu'avec des étudiants en L1 (par exemple, les noms propres - anglais - des décisions judiciaires). Une telle sensibilité, linguistique et culturelle, sera-t-elle évidente dans notre contexte d'observation?

\section{Méthodologie}

\subsection{Le contexte et l'observation}

Cette observation s'est située à l'Université des Sciences sociales Toulouse 1, reconnue mondialement pour l'excellence de la recherche en Sciences économiques, et dont l'école de Gestion (l'Institut d'Administration des Entreprises) vient de signer une charte de qualité certifiant le niveau de ses services. Midi-Pyrénées School of Economics assure depuis plusieurs années l'ensemble de son enseignement en deuxième année de Master $(\mathrm{Bac}+5)$ et en Doctorat en anglais. Les étudiants doivent justifier d'un niveau dans cette langue équivalent au C1 (avancé) sur l'échelle du Cadre européen commun de référence. Mais, depuis 2004-2005, le tiers des cours en première année de Master (M1 = Bac+4) sont enseignés uniquement en anglais. Une certification linguistique n'est pas exigée; les étudiants jugent de leur propre compétence. Ceux qui envisagent de poursuivre un doctorat à Toulouse 1 sont vivement encouragés à suivre les cours en anglais. À l'école de Gestion, un Master en Management international en anglais a été ouvert en 2002. La compétence linguistique, à l'origine évaluée par un test "maison" calqué sur des épreuves standardisées en lecture et en compréhension orale, est depuis 2004 précisée de 
manière plutôt vague : au moyen d'un test américain standardisé (sans précision de score), des notes obtenues en anglais ou d'une attestation.

Notre observation du contexte EMILE a eu lieu en Gestion en 2003 et en Économie en 2005. Le travail ethnographique de Flowerdew et Miller (1995) est un modèle du genre. Réparti sur trois ans, il comprend des questionnaires aux enseignants et aux étudiants ainsi que des journaux personnels, des enregistrements de cours magistraux (CM) avec des entretiens faits avant et après, des notes d'observations et de discussions formelles et informelles, des rapports d'étudiants relatant leurs perceptions, problèmes et stratégies, des groupes de discussion et les documents pédagogiques (notes d'étudiants, manuels, supports polycopiés distribués,) etc.

21 Leur méthodologie servira d'exemple dans de futures études, mais dans le cas présent notre objectif de recherche était plus modeste et, surtout, utilitaire à court terme : avec la mise en place de nouvelles maquettes de Licence et de Master, toute recommandation en matière d'EMILE était - et reste - à faire rapidement. Nous avons vu, ci-dessus, que Teemant et al. (1997) en parlent en termes de négociation en fonction de la reconnaissance institutionnelle des besoins en langue de spécialité. Or, les premières mises en place d'un enseignement en anglais dans le présent contexte ont été préparées sans même évoquer la question avec les enseignants du secteur LANSAD, et les programmes clairement envisagés au moyen de cette langue sans établir d'objectif linguistique explicite.

Il ne s'agit donc pas réellement de CLIL, c'est-à-dire de l'apprentissage (et non de l'enseignement) intégré (et non au moyen) d'un contenu disciplinaire et d'une langue étrangère, mais plutôt d'EMILE (enseignement d'une matière par l'intégration d'une langue étrangère). Hellekjaer et Wilkinson (2003: 90) encouragent à passer du TTFL ( teaching through a foreign language), et donc d'EMILE, à CLIL (content and language integrated learning). Il nous semble qu'un nouveau sigle en français traduirait de manière plus fidèle ce modèle optimal, réellement double, mis en valeur dans la littérature: AIMEL Apprentissage (et non enseignement) Intégré (et non par ou au moyen) d'une Matière et d'une Langue.

Les programmes à Toulouse 1 ont évidemment le mérite d'exister et la logique est clairement celle de l'excellence à frontières ouvertes (et non d'une économie d'échelle en supprimant un enseignement en anglais; dans ces filières, et à ces niveaux d'études, il n'en existait pas). Notre travail, entrepris dans le cadre d'une mission de réflexion sur les langues et la réforme LMD, a visé une prise de conscience de la part des responsables institutionnels de la spécificité du LANSAD dans ce nouveau cadre qu'est l'AIMEL.

Pour cette observation pilote, nous nous sommes d'abord contentée de plusieurs discussions avec une des enseignantes en Management international - une collègue avec qui nous travaillons en anglais de spécialité depuis plusieurs années (Taillefer 2002) et avec qui il existe un climat de confiance - avant et après l'observation directe d'un seul $\mathrm{CM}$ en 2003. Nous avons pu également consulter les évaluations faites par les étudiants à la fin de l'année universitaire. En Sciences économiques, nous avons interviewé huit enseignants (sur onze) ayant fait cours en anglais en 2004-2005 pour la première fois à Toulouse 1 au niveau Bac+4. L'évaluation remplie par les étudiants en Master M1 n'a pas cerné les enjeux réellement posés par cette initiative ; encore une fois, les enseignants de langue n'ont pas été impliqués. Ce champ réduit d'investigations offre néanmoins des renseignements sur d'éventuelles difficultés linguistiques, interculturelles et 
pédagogiques. Il sera davantage question du cas de Management international, le recueil des témoignages des enseignants en Sciences économiques étant en cours au moment où nous écrivons.

\subsection{Les acteurs : étudiants et enseignants}

Lors d'une étude préalable, l'auteure a sondé la représentation chez les enseignants du niveau cible nécessaire pour les diplômés à $\mathrm{Bac}+5$ dans chaque compétence langagière. $\mathrm{Si}$ un niveau B2-C1 (intermédiaire fort-avancé) est jugé minimal en lecture, B1-B2 (intermédiaire faible-fort) a semblé suffire pour les autres compétences (Taillefer 2004b : 114). Les étudiants en M1 en Sciences économiques sont-ils à la hauteur? La même enquête a comporté un audit calibré des compétences réceptives selon l'échelle européenne. Pour la lecture $54,5 \%$ d'un échantillon représentatif d'étudiants étaient A2 (élémentaire), 30,7\% B1 et seulement 9,9\% B2. Pour la compréhension orale 57,4\% A2, $29,7 \%$ B1 et 5,9\% B2. En outre, Taillefer (2004a) a relevé de nombreuses difficultés chez cette même population dans la lecture d'articles scientifiques en anglais : des problèmes grammaticaux, syntaxiques, lexicaux, stratégiques et métacognitifs. Et si $73,3 \%$ d'un échantillon représentatif de leurs enseignants voient un lien « complémentaire » entre la lecture universitaire et la réussite aux examens, $20 \%$ n'y voient qu'un lien faible! Parmi un échantillon représentatif des étudiants (Bac+3 et $\mathrm{Bac}+4)$, ils sont $45,3 \%$ à y voir un intérêt « complémentaire ", mais 37,7 \% un lien faible (Taillefer, à paraitre). Du moins au niveau M1, il est difficile de conclure à une culture d'investissement personnel et de construction de savoir en Sciences économiques.

Le même audit linguistique a été mené chez 502 étudiants en Gestion $(\mathrm{Bac}+3$ à $\mathrm{Bac}+5)$, théoriquement représentatifs du vivier dont sont issus les étudiants de Management international. Meilleurs que les étudiants en Économie, cet échantillon était 39,6\% A2, $48,4 \%$ B1 mais seulement 7,4 \% B2 pour la lecture, et pour la compréhension orale $28,4 \%$ A2, 37,1 \% B1 et 24,6 \% B2. Un enseignement interactif est encouragé dans ce programme, évalué de manière plus variée qu'en Sciences économiques. Une dissertation est obligatoire, rendue en anglais ou en français, mais il est précisé que la qualité linguistique n'est pas prise en compte.

Quant aux neuf enseignants impliqués dans cette étude, tous sauf un sont francophones (dont trois mènent leur carrière plutôt hors de France). Le seul anglophone est parfaitement bilingue. Tous étaient volontaires pour enseigner en anglais et trois d'entre eux ont suivi des cours d'anglais ou ont séjourné dans un pays anglophone pour se préparer à ce nouveau défi. Enfin, ce groupe était composé de quelques jeunes enseignants et d'une majorité de collègues plus âgés, dont certains approchaient l'âge de la retraite.

Leur enseignement était magistral dans tous les cas, mais si le cours de Management international est limité par sélection à environ 30 personnes et permet une interaction, les cours en M1 Économie sont ouverts à l'ensemble de la promotion (environ 350). Finalement, ces cours en anglais ont attiré entre 25 et 35 étudiants chacun, et tous les enseignants ont pu adopter un modèle plutôt interactif. Alors que $28 \%$ des étudiants en Économie viennent de pays étrangers (Scandinavie, Maghreb, Europe de l'est, Chine), la très grande majorité des étudiants ayant suivi les cours en anglais étaient étrangers et non anglophones; les mêmes cours enseignés en français en 2003 ont attiré environ 120-130 étudiants chacun. En Management international, le groupe était composé pour moitié 
d'étrangers - originaires de presque tous les continents - et pour le reste de Français. Le cours en Management international, d'une durée de huit heures seulement, était un aperçu de la comptabilité internationale et des nouvelles normes européennes. Les étudiants avaient des niveaux de spécialité comptable très hétérogènes. En Économie, il s'agissait de plusieurs matières semestrielles, d'une durée de 37,5 heures, destinées à des étudiants spécialistes (autant que faire se peut au niveau $\mathrm{Bac}+4$ ).

\section{Observations}

\subsection{De la part de l'enseignante de comptabilité internationale} aux États-Unis. différentes?

Elle fait part d'emblée des perspectives multiples de l'EMILE (puisqu'il ne s'agit pas réellement de l'AIMEL), dont parle Wolff (2003) et du rapport intrinsèque entre une discipline et son vocabulaire. Pour elle, enseigner en anglais l'oblige à vérifier le cadre conceptuel de sa discipline - à revenir à la base - à travers le vocabulaire spécialisé. Cela lui a demandé une recherche sur les connotations polysémiques entre le français et l'anglais. "Les mots n'ont pas le même sens dans chaque langue ", dit-elle : il s'agit de la langue plus l'esprit plus le sens. À la base de ces différences se trouvent les différents modèles comptables français et anglo-saxons, inspirés des traditions économiques et juridiques respectives.

Elle donne l'exemple du mot-concept «bilan», exemple frappant car c'est la première chose dite aux étudiants. En français, le terme veut dire "photographie du patrimoine » et tous les apprenants le comprennent sans autre explication, étant familiers avec le sens de "patrimoine » dans la société française (patrimoine familial, national, culturel). C'est bien plus qu'un «balance sheet " au sens littéral, mais comment expliquer le sens de "bilan » à des non Français? Un deuxième exemple, « earning ", se traduit généralement par « résultat » et « revenu », alors qu'en comptabilité française, les termes ne sont pas synonymes. Ou encore, le principe comptable de « prudence » en français se traduit par le même mot au Royaume-Uni, mais par « conservatisme », avec une connotation négative,

Troublée par l'origine et les connotations du mot «transparence ", concept-clé dans la comptabilité internationale, elle voulait savoir depuis quand le terme était devenu courant dans la littérature anglo-saxonne. En posant la question à un spécialiste anglais reconnu en comptabilité internationale, les réponses sont revenues de plusieurs endroits dans le monde, en allant des années 1990 à celle d'une utilisation plutôt européenne (« bruxelloise »), et à... une traduction d'un concept français !

2 Pleinement consciente du poids du vocabulaire disciplinaire, elle l'est aussi au niveau des écueils pédagogiques propres à l'environnement d'EMILE. Ainsi, elle se pose la question de savoir où en est l'étudiant dans son champ polysémique dans les exemples qu'elle relève (la valeur d'un mot). Comprend-il ce qu'elle veut dire? Dans une salle de classe franco-française, son indicateur est la dilatation des pupilles, les étudiants s'exprimant rarement en cas de difficulté. Comment jauger dans un groupe d'étudiants de cultures

Enfin, sur le défi inhérent à la situation, elle savait qu'il y aurait un prix à payer pour enseigner en anglais, mais elle savait aussi qu'elle en profiterait. La langue de spécialité est connue de cette chercheuse active; elle a donc suivi des cours d'anglais général 
pendant deux ans. Néanmoins, elle estimait à 30 heures la préparation d'une heure de cours en anglais. Les difficultés se trouvaient, comme indiqué plus haut, au niveau de la clarification des concepts (et donc du vocabulaire qui les véhicule), mais surtout au niveau de la compétence pragmatique. Elle ressentait comme nécessaire de répéter son cours à plusieurs reprises pour que la langue de la présentation et de l'interaction en cours lui vienne automatiquement, surtout dans la mesure où elle s'était engagée à respecter le contrat (moral) de se tenir au programme distribué en début d'année (contrainte auto-imposée ou non par ailleurs). Ses notes de cours, montrées en confiance, étaient beaucoup plus élaborées (surlignées de couleurs différentes, clairement présentées, etc.) que lorsqu'elle donne un $\mathrm{CM}$ dans sa matière en français. Malgré ses vaillants efforts, elle exprimait le "reduced personality syndrome ", ce terme de linguistique appliquée qui décrit le fait de ne pas pouvoir s'exprimer en L2 de manière aussi riche qu'en L1.

\subsection{Nos observations en comptabilité internationale} performance de cette enseignante très similaire à ce qu'elle fait dans ses cours en français (auxquels nous avons assisté à plusieurs reprises). Elle vise dans les deux cas une compréhension aussi large que possible et cherche à la vérifier. La principale différence pédagogique était, effectivement, un style moins vivant et spontané en anglais, accompagné d'une lenteur relative et de silences, non dans le débit de la parole, mais dans les liaisons interphrastiques ou entre deux idées. Elle avait quelques difficultés pour comprendre les questions des étudiants. C'est l'exemple même de la difficulté de " conversational fluency » qu'évoquent Teemant et al $(1997: 316)$ et Hellekjaer et Westergaard (2003: 75).

correct, le vocabulaire académique recherché («enhance») et les phrases, comme le discours, bien construits et liés. Elle a parlé sans lire (alors que certains collègues dans la même université dictent leurs cours en français). dont aucune n'a nui à la compréhension du message : accent tonique mal placé (« assets, development, indexed, purpose »), phonème prononcé ou mal prononcé («debtors, method, cultural, audit, higher »), temps verbaux (« (has) begun, your colleague earn(ed)»), vocabulaire ( the Plan Goering was left [dropped] in $1945 »)$, prépositions (« some of shares »), pronoms (" to protect you [yourself] »). Égale à elle-même, l'enseignante voulait mes corrections après le cours, comme nous avons l'habitude de faire lors de cours communs.

Enfin, nous avons pu consulter les évaluations du programme faites par les étudiants. Il y avait, en fait, peu de commentaires : deux étudiants la trouvaient bien et claire, un seul a noté que le cours pouvait être "plus attrayant» et que son niveau d'anglais était «à revoir ». D'éventuelles difficultés linguistiques du côté des étudiants n'ont pas été évoquées.

\subsection{Enseignants en Sciences économiques}

Ces interviews étaient destinées, d'abord, à faire accepter le principe qu'un intérêt de la part des enseignants du LANSAD ne représente ni une « inspection » ou un jugement sur 
la compétence linguistique ou pédagogique des enseignants d'économie, ni une manière déguisée de s'imposer dans le développement des cursus. Au niveau de la direction de l'UFR, si une hésitation a été exprimée sur l'interprétation possible de la démarche auprès des collègues concernés, l'aval a été accordé (et d'ailleurs a donné lieu à une analyse des besoins professionnels en langues étrangères pour l'ensemble du personnel enseignant et administratif de l'université). Nous avons donc mené ces interviews dans un esprit de négociation diplomatique, en essayant d'établir un climat de confiance et en évoquant la possibilité d'un soutien auprès du Département des langues. Un enseignement de Formation continue destiné aux enseignants (et au personnel administratif) a d'ailleurs existé mais a été supprimé il y a deux ans; certains enseignants interviewés s'en sont plaints.

Le résultat de cette démarche porte déjà ses fruits, aussi bien sur le plan psychologique que scientifique. L'intérêt d'une collègue de LANSAD non seulement n'a pas posé de problème, mais au contraire a été accueilli avec plaisir (l'interview était un moment privilégié de partage et d'intérêt professionnel réciproque), ce qui nous a permis de cerner les questions des enjeux et des risques de l'EMILE. Deux collègues nous ont cordialement invitée à assister à leurs cours l'an prochain.

Nous avons évoqué avec ces huit collègues la composition démographique de leur groupe d'étudiants, les difficultés qu'auraient pu manifester leurs étudiants, et leurs propres réactions sur les questions linguistiques, interculturelles et pédagogiques. D’abord, comme il a déjà été noté, ce sont très majoritairement les étudiants étrangers qui ont choisi de suivre les cours en anglais en M1, mais nous n'avons pas le moyen de vérifier leur motivation ni celle des étudiants, surtout français, qui n'ont pas choisi ces cours. Sur le plan linguistique, Hellekjaer et Westergaard (2003: 73) ont rapporté ne pas avoir trouvé de difficultés linguistiques sérieuses («dramatic») chez les apprenants dans la plupart des 58 programmes explorés en Scandinavie, avec toutefois davantage de problèmes là où il n'y avait pas de sélection linguistique préalable et chez les étudiants les plus jeunes. Tous les enseignants ont affirmé qu'il n'y avait pas de problème linguistique apparent chez les étudiants, à part le cas de quelques étudiants français. L'auto-sélection linguistique a donc joué le rôle de filtre. Les rares fois où des questions de langue se sont posées, les enseignants ont dit avoir essayé d'y remédier et d'y répondre, en anglais. La traduction a été très rarement utilisée.

En revanche, trois de ces collègues français - ceux ayant le profil le moins international ont librement parlé de leurs propres difficultés linguistiques en termes, comme l'enseignante de comptabilité, de manque d'aisance "conversationnelle» et donc de rapports humains moins satisfaisants, de se sentir moins clairs et moins efficaces qu'en français, d'une charge cognitive plus lourde (à la recherche de synonymes, d'amorces de phrases et de paraphrases), d'une fatigue sur la durée (un cours de trois heures) et enfin d'un certain stress, voire d'anxiété. Un des enseignants les plus expérimentés a fait part d'un manque de confiance au départ, traduit par l'impression que, en anglais, il avait besoin de "conquérir» le public, dans la mesure où son niveau d'anglais à l'oral était moins élevé que celui de ses étudiants. Au moyen d'un « contrat » (accepter des questions en français, mais les traduire et y répondre en anglais), il a pu établir un climat de complicité avec les étudiants, comme il a l'habitude de le faire en français.

Malgré ces difficultés, tous ces enseignants volontaires étaient contents de leurs efforts, ont senti une progression et un gain de facilité, et celle qui est sans doute la plus faible en 
anglais - et donc la plus courageuse - a dit attendre l'an prochain pour réaliser une meilleure "performance ». Trois des collègues ont comparé les difficultés du démarrage du semestre au fait de... reprendre le vélo au printemps. Ayant reçu ce genre de message, les collègues de LANSAD pourraient, par exemple, proposer quelques séances de "gym conviviale » au début de chaque semestre. Le sentiment de l'enseignante la plus fragile linguistiquement était en effet que si l'université souhaite développer ce type d'enseignement, il faut qu'elle y mette les moyens.

Les réflexions interculturelles et pédagogiques se sont souvent recoupées. Tous les collègues ont trouvé les étudiants étrangers (ERASMUS ou inscrits en formation diplômante française) très motivés, voulant s'investir nettement plus que les étudiants «ordinaires» et à la recherche de la lecture (alors que nous avons vu plus haut la faiblesse du lien perçu entre lire et réussir par ces derniers). La plupart des abandons constatés étaient ceux de Français. Selon un enseignant (d'origine belge), les étudiants « ordinaires » appliquent la loi du moindre effort, évitant aussi bien les cours complexes que les cours en anglais. Ces huit enseignants étaient tous très satisfaits du niveau scientifique atteint et, lorsqu'une comparaison était possible avec le même cours enseigné en français l'année précédente, ils ont trouvé le niveau plus élevé en 2004-2005. Trois enseignants ont dit qu'ils comptaient fixer des objectifs plus élevés l'année prochaine (notamment une nouvelle recrue à qui ses collègues avaient suggéré qu'il valait mieux prévoir de dicter ses cours).

45 Pour ces professeurs, si enseigner en anglais a demandé davantage de temps de préparation pour certains (mais moins pour d'autres obligés de trouver le vocabulaire approprié dans leur L1 lorsqu'ils enseignent en français), la plupart ont trouvé qu'ils ont mené le cours de la même manière qu'ils l'auraient fait en français. Certains ont dit utiliser davantage de supports visuels, et un a "osé » exiger un manuel en anglais (impensable, a-t-il dit, dans un cours en français).

46 Enfin deux enseignants ont fait des observations particulièrement pertinentes sur l'imbrication de la langue étrangère et de l'interculturel. Le collègue le plus jeune, et le seul à avoir eu un nombre plus important d'étudiants français (le cours étant obligatoire pour eux), a été tout de suite conscient du fait qu'ils comprenaient mal : d'abord parce qu'ils étaient les seuls à copier les transparents "à outrance, au lieu d'écouter " et qu'ensuite leurs (rares) questions étaient généralement peu pertinentes. Il a regretté qu'il n'y ait pas eu d'évaluation officielle du programme.

47 Le seul enseignant anglophone, lui-même bilingue et biculturel, a parlé du défi pour déterminer si l'origine d'une difficulté de compréhension chez les étudiants relève de la langue, du concept, ou des deux. C'est également le seul enseignant, d'une part, à avoir physiquement demandé à des étudiants «bavards » de sortir de son cours, et d'autre part, à avoir rencontré le plagiat. Comme l'évaluation de son cours s'est faite uniquement au moyen d'un mémoire (pratique peu courante, comme nous l'avons vu ci-dessus), il a exigé une déclaration sur l'honneur. La réponse institutionnelle dans cette affaire (Conseil de discipline) déterminera, en partie, s'il continuera ainsi. Enfin, cet enseignant a également soulevé une question qui reste à vérifier : puisque le niveau scientifique dans ces cours en anglais semble généralement meilleur que dans ceux enseignés en français, y aura-t-il une cristallisation d'un enseignement à deux vitesses, en plus à caractère ethnique évident? Si oui, est-ce que les étudiants « ordinaires » seront motivés pour améliorer leur niveau en anglais ? Ou, au contraire, y aura-t-il une «fracture linguistique », ou pire, une inflation statistique des notes plus faibles par souci de traitement égal? 


\section{Discussion et implications}

EMILE et non d'AIMEL (Apprentissage Intégrée d'une Matière et d'une Langue). La recherche appliquée indique pourtant cette deuxième voie comme la meilleure. Pour y arriver, il faut prendre en compte les questions de curriculum, d'approche pédagogique et d'évaluation, de formation professionnelle et de soutien aux apprenants. C'est ainsi que nous proposons, à la base, une prise de conscience accrue, de la part des responsables et des enseignants, des enjeux et des risques de ces programmes, mais aussi des bénéfices à en tirer (notamment en objectifs linguistiques, non laissés au hasard). Cette prise de conscience résultera d'une collaboration institutionnelle reconnue entre le Département des langues et les différentes disciplines (Facultés, Instituts, etc.). Les bases ont été jetées en 2004-2005, en procédant de manière "bottom up », de collègue à collègue, dans une démarche non menaçante et axée sur le partage et le soutien.

51 Ainsi, en réponse aux dernières questions de recherche et dans un cadre décloisonné, nous proposons :

- une évaluation formative chez les étudiants suivant les cours en anglais pour identifier leurs difficultés (comme nous l'avons fait pour leurs enseignants). Ceci permettrait de cerner la question délicate d'une « fracture linguistique » ou d'une demande de mise à niveau.

- la mise en place d'un soutien pour les enseignants volontaires souhaitant relever ce défi. Une analyse des besoins à large échelle est actuellement en cours, mais un tel soutien pourrait prendre diverses formes: travail encadré ponctuel, suivi ou intensif sur le métalangage de l'enseignement, sur la langue "conviviale ", sur un thème ou une tâche donnés, travail en autonomie guidée, etc. Cela serait l'occasion idéale d'évoquer et d'optimiser le potentiel des enseignants disciplinaires en tant que références linguistiques (LANSAD «à l'envers »). Nos échanges nous ont déjà permis de semer des graines (des « tuyaux » proposés à certains collègues). 
- une réflexion sur la certification linguistique exigée dans l'octroi du titre de Master, où le candidat est censé "maîtriser» au moins une langue vivante étrangère. C'est aux universités de définir leur conception de cette maîtrise, les jurys étant souverains en la matière. Le Cadre européen commun de référence en sera la base, mais des efforts de formation professionnelle sont à faire pour que les enseignants de LANSAD s'approprient cet outil d'apprentissage, d'enseignement et d'évaluation. des bases de la réforme LMD favorisant la mobilité internationale des étudiants, préconise, d'une part, une sélection à l'entrée en M1 (jusqu'alors réservée à l'entrée en M2) et, d'autre part, une augmentation de $50 \%$ du nombre de diplômés. Cette volonté se traduirait par une nette augmentation de la demande des cours intégrant contenu et langue étrangère et insisterait sur la notion de certification.

Comme l'affirment Marsh et al. (2001), la réussite à long terme de l'apprentissage intégré d'une matière et d'une langue (CLIL, AIMEL) ne sera assurée - en supposant déjà l'excellence disciplinaire - qu'en fonction de la reconnaissance des enjeux linguistiques et interculturels, et de la sensibilisation des enseignants impliqués. Ce rôle pourrait revenir aux anglicistes spécialistes de LANSAD, ayant posé ces questions de recherche et de pratique depuis longtemps, et ayant joué le rôle de médiateurs depuis toujours. Un tel travail de collaboration exigera l'engagement de l'établissement; nous relevons, dans le contexte français, le défi qui a été clairement exprimé par Flowerdew et Miller ailleurs :

Effective communication in the cross-cultural lecture theatre comes at a high price; are institutions aware of - and willing to - make the necessary investment? (1995 : 370)

\section{BIBLIOGRAPHIE}

Conseil de l'Europe, 2001. Cadre européen commun de référence pour les langues : apprendre, enseigner, évaluer <http://culture2.coe.int/portfolio>.

Dudley-Evans, T. \& M. J. St. John. 1998. Developments in English for Specific Purposes: A Multidisciplinary Approach. Cambridge : Cambridge University Press.

Flowerdew, J. \& L. Miller. 1995. « On the notion of culture in L2 lectures ». TESOL Quarterly 29, 345-373.

Flowerdew, J. \& L. Miller. 1996. « Lectures in a second language: Notes towards a cultural grammar ». English for Specific Purposes15, 121-140.

Grabe, W. \& F. Stoller. 1997. «Content-based instruction: Research foundations ». In Snow, M. \& D. M. Brinton (dir.), The Content-Based Classroom. White Plains, NY : Addison-Wesley Longman, 78-94.

Hellekjaer, G. O. \& M. R. Westergaard. 2003. « An exploratory survey of content learning through English at nordic universities ». In Van Leeuwen, C. \& R. Wilkinson (dir.), Multilingual Approaches in University Education: Challenges and Practices. Nijmegen : Uitgeveerij Valkhof Pers \& Talencentrum Universiteit Maastricht. 
Hellekjaer, G. O. \& \& R. Wilkinson. 2003. « Trends in content learning through English at universities: A critical reflection ». In Van Leeuwen, C. \& R. Wilkinson (dir.), Multilingual Approaches in University Education: Challenges and Practices. Nijmegen : Uitgeveerij Valkhof Pers \& Talencentrum Universiteit Maastricht.

Hofstede, G. 1991. Cultures and Organization: Software of the Mind. Londres : McGraw-Hill.

Isani, S. \& D. Andreolle. 2001. « Langue de la culture et culture de la langue : la dimension didactique de la culture des langues de spécialité.» In Mémet, M. et M. Petit (dir.) L'anglais de spécialité en France, Mélanges en l'honneur de Michel Perrin. Bordeaux : GERAS.

Kaplan, R. 1966. « Cultural thought patterns in intercultural education ». Language Learning 16, 1-20.

Kaplan, R. 1995. « Contrastive rhetoric ». TESOL France Journal 1, 28-58.

Kurtàn, Z. 2003. « Teacher training for English-medium instruction ». In Van Leeuwen, C. \& R. Wilkinson (dir.), Multilingual Approaches in University Education: Challenges and Practices. Nijmegen : Uitgeveerij Valkhof Pers \& Talencentrum Universiteit Maastricht.

Marsh, D., B. Marsland, B. \& K. Stenberg. 2001. Integrating Competencies for Working Life. Jyväskylä, Finlande : Unicom, University of Jyväskylä.

Mémet, M. 2001. «Bref historique de l'enseignement et de la recherche en anglais de spécialité en France : de l'anglais pour non-spécialistes à l'anglistique du secteur LANSAD ». In Mémet, M. et M. Petit (dir.), L'anglais de spécialité en France, Mélanges en l'honneur de Michel Perrin. Bordeaux : GERAS.

Mémet, M. 2003. «L'enseignement à contenu intégré augmente la motivation pour l'apprentissage de la langue : Vrai ou Faux ? Étude portant sur des cours d'anglais de spécialité en médiation culturelle ». ASp 39-40, 131-142.

Orlac'h, P. 2005 « Les barrières linguistiques sont-elles un frein à l'accueil d'étudiants étrangers ? se demande La Lettre d'Égide d'avril 2005 ». L'Agence éducation emploi formation (L'AEF), dépêche n ${ }^{\circ} 52304 \mathrm{du} 18$ avril.

Stoller, F. \& W. Grabe. 1997. « A Six-T’s approach to content-based instruction ». In Snow, M. \& D. M. Brinton (dir.), The Content-Based Classroom. White Plains, NY : Addison-Wesley Longman.

Swales, J. M. 1990. Genre Analysis. Cambridge : Cambridge University Press.

Taillefer, G. (à paraître). « Reading For Academic Purposes: The Literacy Practices of British, French and Spanish Law and Economics Students as Background for Study Abroad ». Journal of Research in Reading.

Taillefer, G. 2004a. « Lire en anglais à l'université : étude de cas d'étudiants en Maîtrise de Sciences économiques confrontés à l'article scientifique ». Les Cahiers de l'APLIUT 23/3, 76-90.

Taillefer, G. 2004b. « Une analyse critériée des besoins linguistiques dans l'enseignement universitaire des Sciences économiques ». ASp 43-44, 107-124.

Teemant, A., E. Bernhardt \& M. Rodrigues-Muñoz. 1997. « Collaborating with content-area teachers: What we need to share ». In Snow, M. \& D. M. Brinton (dir.), The Content-Based Classroom. White Plains, NY : Addison-Wesley Longman, 311-318.

Ulijn, M. \& F. Salager-Meyer. 1998. « The Professional Reader and the Text: Insights from L2 Research ». Journal of Research in Reading 21, 79-95. 
Valero-Garcés, C. 1996. «Contrastive ESP rhetoric: Metatext in Spanish-English economics texts ». English for Specific Purposes 15, 279-294.

Wolff, D. 2003. « Integrating language and content in the language classroom: Are transfer of knowledge and of language ensured? ». ASp 41-42, 35-46.

\section{NOTES}

1. Il s'agit d'un entretien personnel avec Stewart Field de l'Université de Cardiff (Law School).

\section{RÉSUMÉS}

À l'instar des programmes destinés à favoriser la mobilité des étudiants dans d'autres pays européens, l'enseignement en France d'une matière disciplinaire en langue étrangère se développe. Si la recherche souligne le bien-fondé de cette approche intégrée, elle met en relief également les écueils dus, d'une part, aux divergences linguistiques, interculturelles et pédagogiques entre enseignants et étudiants et, d'autre part, au niveau de reconnaissance institutionnelle de ces facteurs. Nous avons voulu observer la mise en œuvre d'un tel enseignement dans une université de sciences sociales pour identifier d'éventuelles difficultés et pour encourager une prise de conscience institutionnelle. Neuf enseignants de Gestion et des Sciences économiques ont été interviewés dans cette démarche pilote. Il semble que, dans les conditions actuelles, cette mise en place soit réussie, mais des risques ont été mis à jour ; ils sont liés à l'envergure du programme, à la reconnaissance de difficultés linguistiques chez les différents acteurs, et au degré réel d'intégration d'objectifs d'apprentissage disciplinaires et linguistiques. Cet enseignement intégré sera fructueux si ces questions sont prises en compte.

Like programmes in other European countries aimed at encouraging study abroad, teaching subject matters in a foreign language is developing in France. Research has shown the benefits of this integrated approach, but it also points out possible pitfalls due to linguistic, intercultural and pedagogical divergences between teachers and students, and to the extent to which these issues are recognized by the institution. Our aim was to observe the initial implementation of this approach in a French university specializing in social sciences in order to pinpoint possible problems and to encourage institutional awareness of such problems. In this pilot study, nine Management and Economics teachers were interviewed. Within the present limited context, the undertaking seems successful, but risks are identified linked to the scale of the programme, to the recognition of language problems in students and teachers, and to the level of true integration of both content and language learning objectives. Fruitful development of this integrated approach will depend on consideration of these issues. 
INDEX

Mots-clés : compétence interculturelle, compétence linguistique, ECI, EMILE, objectifs, pédagogie, reconnaissance institutionnelle

Keywords : CLIL, institutional recognition, intercultural competence, linguistic competence, objectives, pedagogy

\section{AUTEUR}

\section{GAIL TAILLEFER}

Gail Taillefer est maître de conférences d'anglais. Ses travaux et son enseignement portent sur le transfert des compétences de la L1 vers la L2 dans la compréhension de texte, la compréhension auditive et l'expression orale, ainsi que dans le domaine interculturel. L'aspect stratégique de ces questions et leur mise en œuvre au niveau institutionnel sont au cœur de sa démarche.

gail.taillefer@univ-tlse1.fr 